\title{
Bone Marrow Examinations: Findings from Four Year Retrospective Single Centre Review
}

M. MIZANUR RAHMAN ${ }^{1}$, M. A. KHAN ${ }^{1}$, SALMAAFROSE ${ }^{1}$, TASMI N ARA ${ }^{1}$, SIRAJUL ISLAM ${ }^{1}$, AKHI L RANJ AN BI SWAS ${ }^{1}$

\begin{abstract}
Background: Bone marrow examination is used for diagnosis of both haematological and non-haematological diseases.

Materials and methods: In this retrospective study data from the registry of department of haematolgoy, Dhaka medical college was analyzed. Total 2340 bone marrow was reported for the period of 2006-2009.

Results: The most prevalent disease is acute leukaemia (1022, 43.67\%) followed by non-specific reactive and normal marrow (495, $21.15 \%)$.

Conclusion: The study indicates that the indication for bone marrow is not optimum which is related with patient's poor economic condition and physicians' limited exposure to haematology. Sole reliability on Rowmanwsky stain is the major limitations of the bone marrow reports. Optimization of indication, reporting and further supplementary investigation is needed for further improvement.
\end{abstract}

Keyword: Bone Marrow Examination, Laboratory Techniques and Procedures, Bangladesh

\section{Introduction}

Bone marrow examination is an important diagnostic tool for both haematological and non-haematological diseases. In bone marrow examination, along with history, clinical examination and peripheral blood film; a morphological diagnosis was made. For morphological examination leishmen stain, cytochemistry and iron stain can be done. ${ }^{1}$

This is an invasive procedure which has some amount of physical and psychological trauma with potential risk of complications. A number of aspirations do not bring adequate material. In Bangladesh, bone marrow examination reports are exclusively done by the haematologists.

In this study we tried to find out the disease prevalence, limitations and rationality of the bone marrow examination in one tertiary center.

\section{Materials and methods:}

This is a retrospective observational registry based study from the department of haematology in Dhaka Medical College Hospital. This is the largest tertiary hospital in Bangladesh where patients referred from the whole country. Most of the patients reported here from the lower socioeconomic condition representing common population. As a result, though the hospital is located in the capital of the country, it represents a gross picture of health status of Bangladesh.
A hand written registry book of the department of haematology, Dhaka Medical College \& Hospital was kept from the year of its establishment of independent working, 2005. However, the data of 2005 was not included here as it was partial. The entry was done by the haematologists who signed the report. It is common practice to consult with fellow colleagues before finalizing the report. From 2006 to 2009 all the entries were included in this study. There was possibility that some cases were not included into the registry. Cases of aspiration failures were also rarely included in the registry and thus were not included in this study. Patients were from the department of haematology and all other department including both adult and paediatrics. Bone marrow reports made here is based on morphology only with leishmen's stain. Few doubtful cases were further validated with further investigation. Data was analyzed in Microsoft Excel, and presented with tables and graphs. No ethical issue is raised here as data was collected from medical records kept for departmental activities in a public medical college hospital and it does not reveal any personal information which may cause any harm to any subject.

\section{Results:}

A total of 2340 bone marrow reports were included in this study. The prevalence of disease is shown in the Fig 1. This number does not include the aspiration failure cases. The bar chart shows acute leukaemia is the most common

1. Department of Haematology, Dhaka Medical College Hospital

Correspondence: M. Mizanur Rahman, Department of Haematology, Dhaka Medical College Hospital, E-mail: shawon71@yahoo.com 
disease with 1022 cases in total where ALL is the most prevalent disease with 520 cases closely followed by AML 502 cases.

In Table I, the disease prevalence shown by year. The total numbers of reported cases are 377, 527, 712 and 724 for years from 2006 to 2009 respectively. Although the number of total cases in ALL is slightly more than the AML (520 vs. 502) the reverse is true in case of new cases which represent the actual incidence (334 for AML and 284 for ALL). In case of leukemia and multiple myeloma new and old cases is reported separately. Erythroid and myeloid hyperplasia marrow are grouped under reactive marrow. Rare diseases like histoplasmosis, plasma cell leukaemia, pure red cell aplasia etc are grouped under other category.

The percentage of new and old cases in acute leukaemia is further detailed in Fig 2 by a pie diagram (Fig 2).

Table I

Disease prevalence by year

\begin{tabular}{|c|c|c|c|c|c|c|c|}
\hline Diseases & & 2006 & 2007 & 2008 & 2009 & & Total (\%) \\
\hline \multirow[t]{2}{*}{ ALL } & New & 37 & 70 & 100 & 77 & 284 & $520(22.22)$ \\
\hline & Old & 35 & 51 & 69 & 81 & 236 & \\
\hline \multirow[t]{2}{*}{ AML } & New & 49 & 83 & 97 & 105 & 334 & $502(21.45)$ \\
\hline & Old & 37 & 43 & 47 & 41 & 168 & \\
\hline \multirow[t]{2}{*}{ Reactive } & Erythroid & 11 & 33 & 31 & 45 & 120 & 373(15.94) \\
\hline & Myeloid & 51 & 57 & 74 & 71 & 253 & \\
\hline AA & & 51 & 55 & 77 & 74 & & 257(10.98) \\
\hline Mega Нype. & & 36 & 42 & 59 & 41 & & 178(7.61) \\
\hline NAM & & 16 & 21 & 42 & 43 & & $122(5.21)$ \\
\hline LRM & & 16 & 15 & 35 & 26 & & 92(3.93) \\
\hline \multirow[t]{2}{*}{ MM } & New & 9 & 22 & 14 & 24 & 68 & 88(3.76) \\
\hline & Old & 2 & 1 & 3 & 13 & 19 & \\
\hline CML & & 5 & 11 & 23 & 38 & & 77(3.29) \\
\hline MDS & & 7 & 7 & 12 & 21 & & 47(2.01) \\
\hline LD & & 12 & 10 & 15 & 4 & & $41(1.75)$ \\
\hline MA & & 0 & 2 & 7 & 9 & & 18(0.77) \\
\hline Secondaries & & 3 & 4 & 5 & 7 & & $19(0.81)$ \\
\hline Other & & 0 & 0 & 2 & 4 & & $6(0.26)$ \\
\hline Total & & 377 & 527 & 712 & 724 & & $2340(100)$ \\
\hline
\end{tabular}

Abbreviations: ALL- Acute lymphoblastic leukaemia, AML-Acute myeloid leukaemia, AA- Aplastic anaemia,, Mega Hype- Megakaryocytic hyperplasia, NAM- Normal active marrow, LRM- Lympho reticular malignancy, MM- Multiple myeloma, CML- Chronic myeloid leukaemia, MDS- Myelo dysplastic syndrome , LD - Leishmania donovani, MA- Megaloblastic anaemia Secondaries- Bone marrow metastasis. 
Bone Marrw Diagnosis: 2006-2009

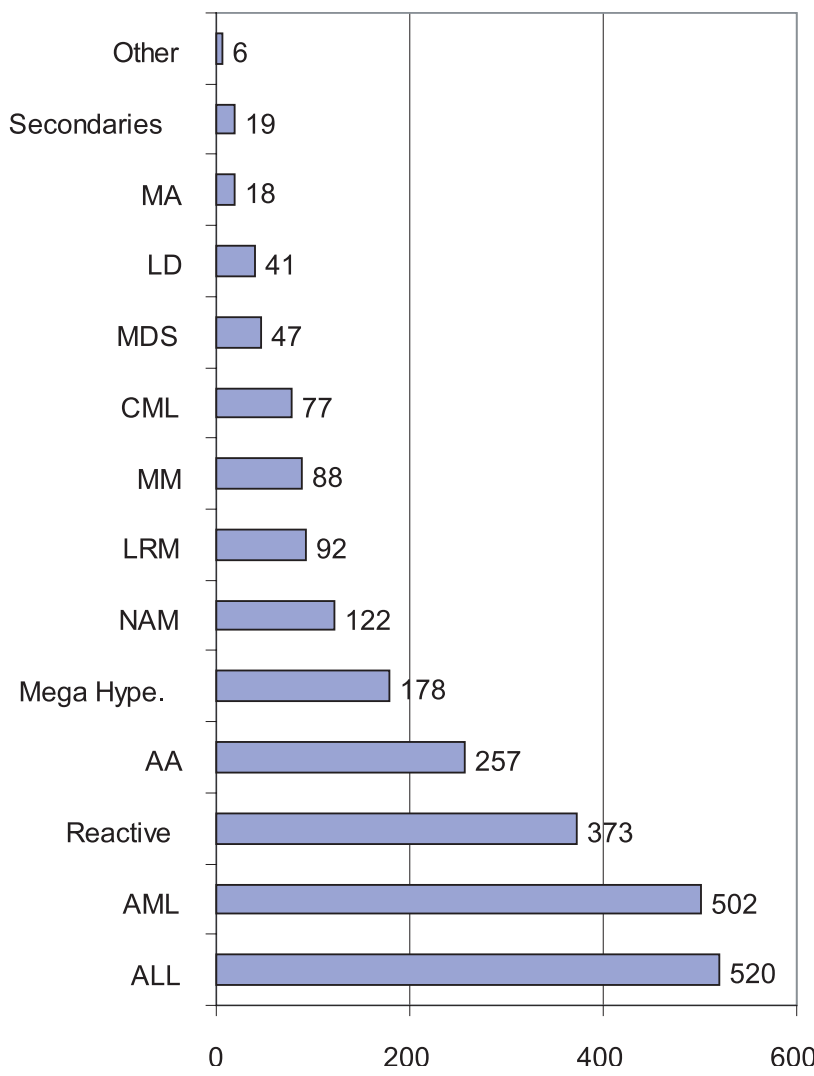

Abbreviations: ALL- Acute lymphoblastic leukaemia, AML- Acute myeloid leukaemia, AA- Aplastic anaemia,, Mega HypeMegakaryocytic hyperplasia, NAM- Normal active marrow, LRMLympho reticular malignancy, MM- Multiple myeloma, CMLChronic myeloid leukaemia, MDS- Myelo dysplastic syndrome, LD - Leishmania donovani, MA- Megaloblastic anaemia Secondaries- Bone marrow metastasis.

Fig.-1: Bone Marrow Diagnosis: 2006-2009

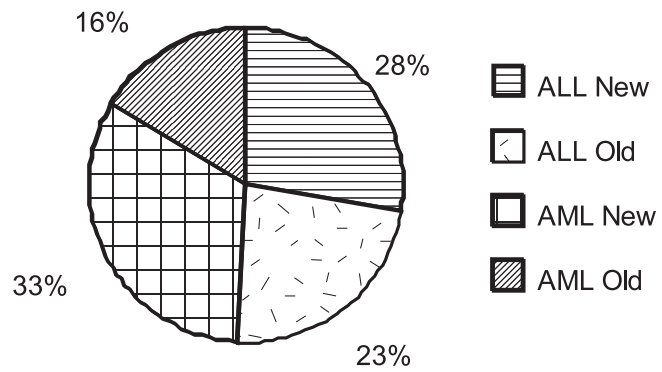

Fig.-2: Reported Cases of Leukaemia, 2006-2009

\section{Discussion}

The increasing number of cases year by year indicated that the bone marrow is a reliable and valid tool for diagnosis in this center. One of the unusual reason for this is, bone marrow examination was often requested or preformed before other costly non-invasive investigations as it is relatively cheap in this center. Over all taka 300 was needed (with some cases done at free of cost) for a test. For example, in case of macrocytic anaemia the Vitamin B12 and folate costs more than Taka 2000.

The bone marrow report was usually reviewed by more than one haematologist most of the time. Clinical correlation was made to make a definite diagnosis. Outside review was done in difficult cases. Major draw back of this diagnosis was that in majority of the cases the morphological findings were not validated by further investigation like cytochemistry, immunophenotype or cytogetics. This is because as most of the patients were not able to bear the further cost. So, haematologists some times are forced to give a conclusive diagnosis even when there is dilemma in morphology. Morphological diagnosis has a certain percentage of limitations. $^{2}$

Acute leukaemia is the leading disease (43.67\%) from a haematology ward as expected. The curious finding is that the new case of AML is more than the ALL (331vs 282) but reverse in the total cases (520 vs. 502). Also old cases are less than the new cases (ALL new cases $28 \%$ vs. ALL old cases $23 \%$ and AML new cases $33 \%$ vs. AML old cases $16 \%)$ This is because poor socio economic conditions often force the haematologist and the family to go for palliative treatment especially in case of AML. So, in many cases after diagnosis further bone marrow was not required. Only exception is ALL data for 2009 where the old cases were more than the new cases. This indicates the improved survival of the ALL patient in this center. Also as bone marrow examination with old patient with palliative option is not absolute, may be many poor patients included in this study may not need this invasive procedure. ${ }^{3}$

Reactive marrow report and normal active marrow (21.15\%) was quite high having the position just after leukaemia. The reason behind it is multiple. NHL stage I and II were the commonest cause. But a large number of cases might not have proper indication for bone marrow. Referral for bone marrow with any kind of cytopenia (i.e. autoimmune haemolytic anaemia) was an example for that. Also there might be cases of MDS- refractory anaemia in this reports as iron stain was not done. Proper referral and further investigation might reduce and change this diagnosis to a significant number.

Megakaryocytic hyperplasia is another interesting finding of this study (7.61\%). Though the clinical indication did not recorded but to the author's experience most of them referred for the confirmation of the diagnosis of ITP. Though it is 
now established that bone marrow is not needed for the diagnosis of ITP. ${ }^{4}$ The trend is declining for the year of 2009 and may decrease further in coming years as the department tries to refuse such kind of referral.

In CML cytogenic study for Philadelphia chromosome was carried out on bone marrow sample. But many patients who could not afford imatinib mesylate and thus were not gone under bone marrow examination. So, the actual number of CML was few more than the number represents here.

\section{Conclusion}

Bone marrow examination is an important diagnostic tool for diagnosis of the haematological and non-haematological diseases. But indication for the examination and the validation of this bone marrow report by further investigation is not optimum. Limitations of this diagnostic tool should be supplemented with other investigations.

\section{Conflict of interest: None}

\section{References}

1. Nguyen D., Diamond L. Approach to bone marrow. In: Diagnostic hematology, a pattern approach. Hodder Arnold, 2006: 270-273.

2. Bunning RD, Maututes E, Flandrin G, et al. Acute myeloid leukaemia not otherwise clategorised. In: Jaffe SE, Harris NL, Stein H, Varidiman JW, eds. Pathology and genetics of tumors of haematopoietic and lymphoid tissues. Lyon: IARC press, 2001: 91-105.

3. British Committee for Standards in Haematology. Guidelines on the management of acute myeloid leukaemia in adults. $\mathrm{Br}$ J Haematol 2006;135:450-474.

4. British Committee for Standards in Haematology task force. Guidelines for the investigation and management of idopathic thrombocytopenic purpura in adults, children and in pregnancy. Br J Haematol 2003;120:574-596. 